Research Article

\title{
Multifeatured Image Retrieval Techniques Based on Partial Differential Equations for Online Shopping
}

\author{
Jiaohui Yu \\ School of Business, Shandong Jianzhu University, Jinan, 250101 Shandong, China \\ Correspondence should be addressed to Jiaohui Yu; yujiaohui19@sdjzu.edu.cn
}

Received 21 August 2021; Accepted 8 September 2021; Published 25 September 2021

Academic Editor: Miaochao Chen

Copyright ( 2021 Jiaohui Yu. This is an open access article distributed under the Creative Commons Attribution License, which permits unrestricted use, distribution, and reproduction in any medium, provided the original work is properly cited.

\begin{abstract}
In today's rapid development of network and multimedia technology, the booming of electronic commerce, users in the network shopping species of images and other multimedia information showing geometric growth, in the face of this situation, how to find the images they need in the vast amount of online shopping images has become an urgent problem to solve. This paper is based on the partial differential equation to do the following research: Based on the partial differential equation is a kind of equation that simulates the human visual perception system to analyze images; based on the summary of the advantages and disadvantages of multifeature image retrieval technology, we propose a multifeature image retrieval technology method based on the partial differential equation to alleviate the indexing imbalance caused by the mismatch of multifeature image retrieval technology distribution. To improve the search speed of the data-dependent locally sensitive hashing algorithm, we propose a query pruning algorithm compatible with the proposed partial differential equation-based multifeature image retrieval technology method, which greatly improves the retrieval speed while ensuring the retrieval accuracy; to implement the data-dependent partial differential equation algorithm, we need to distribute the data set among different operation nodes, and to better achieve better parallelization of operations, we need to measure the similarity between categories, and we achieve the problem of distributing data among various categories in each operation node by introducing a clustering method with constraints. The purpose of this article for image recognition is for better shopping platforms for merchants. This algorithm has trained multiple samples and has data support. The experimental results show that our proposed data set allocation method shows significant advantages over the data set allocation method that does not consider category correlation. However, the image features used in image retrieval systems are often hundreds or even thousands of dimensions, and these features are not only high in dimensionality but also huge in number, which makes image retrieval systems encounter an inevitable problem- "dimensionality disaster." To overcome this problem, scholars have proposed a series of approximate nearest neighbor methods, but multifeature image retrieval techniques based on partial differential equations are more widely used in people's daily life.
\end{abstract}

\section{Introduction}

With the comprehensive and rapid development of ecommerce and the increasing pace of people's lives, online shopping has become fashionable and popular, and the number of online shoppers is constantly growing. Unlike shopping in brick-and-mortar stores, online shoppers are using computers and the Internet to browse the goods they need. Facing numerous shopping sites is like facing a dazzling array of goods, plus the lack of visual and tactile experience in the real shopping process; it is difficult for online shoppers to select the right goods; therefore, a shopping site's product search nowadays, most of the search engines of shopping sites are borrowed from the traditional. The text-based information retrieval technology, from the function and performance, can not meet the user's requirements; the user need is how to get online in the real shopping in the kind of immersive visual and tactile experience, such as the color, shape, texture, and size, and hope in a very short period to get as much information in this regard, to achieve the purpose of buying the required goods. At this time, a content-based, multimedia information retrieval tool 
that incorporates images and text for shopping website engine configuration is important. The development of partial differential equation-based image retrieval technology can facilitate people to retrieve the part they need for the products appearing on the Internet, which shows that visual images are an important vehicle for individual information interaction. Since the "information age," the application and demand of information data have entered a booming stage, and information interaction through sensor technology has become an important way to obtain and disseminate data and information. Image recognition technology is particularly important for people's online shopping experience. This article focuses on algorithms that improve its accuracy and uses the platform to bring people a better life experience.

There are two ways to retrieve image information in the database, namely, full-text indexing and keyword indexing. The basic step is to establish full-text indexing and keyword indexing based on the automatic collection and annotation of web page information as an important component of the search engine. Full-text indexing is to use all the text information of the web page where the image is located as the annotation of the image, and any text information on the web page is considered to be related to the image; this image annotation has a high search rate, but the search accuracy rate is very low, based on key. The indexing of words uses several keywords to represent the image information, which is generally obtained from the seller's description of the image of the product, which has a high search accuracy in comparison but a low search completeness literature [1].

E-commerce shopping website has its uniqueness compared with general websites, that is, to present the information of goods directly; they all use pictures as information carriers. Commodity information itself has its characteristics, such as the sensitivity to time and dependence on color, like clothes, shoes, etc., but the seasonal changes of dependent goods, resulting in the rapid number of images. With the growth and the rapid increase in the number of image information, the use of the above two retrieval methods has the following problems: (1) the diversity of product information classification by the sellers of goods. Salespersons are the publishers of online information, and they cannot classify the information according to the relevant taxonomy but label the image files according to their understanding and preference, which is highly subjective, and thus, there is bound to be a diversity of citation terms, which increases the scope of retrieval; (2) ambiguity in consumers' understanding of commodity information labels, i.e., due to differences in personal perception, different people treat the same image; (3) the ambiguity of consumers' understanding of product information labels, i.e., due to differences in individual perception, different people may have a different understanding of the same image; (4) the different classifications of visually dependent product types, i.e., for this kind of visually dependent product, there is a difference in the classification of product types. Goods, different angles of division will lead to different types of belonging; and (5) incompleteness of commodity information labeling, i.e., the text labeling of goods does not fully reflect the content of the imaging literature [2].
The use of image retrieval technology to integrate the feature images captured by different sensors can achieve a comprehensive representation of the target scene information, which is more convenient for the system to complete the task accurately and effectively. At present, image retrieval technology is widely used in huge number of fields such as military, medical, transportation, aviation, and industrial vision and has very important application value for national defense and people's livelihood, scientific and technological innovation, and economic construction. With the increasing standard of living, people's online shopping needs are increasing, especially the image retrieval technology based on partial differential equations is used in various aspects, especially online shopping, which allows people to quickly find the products they need.

\section{Related Work}

Since the 20th century, the rapid development of electronic computer technology has pushed mankind into the electronic information era; electrical automation, computer applications, sensor technology, and information processing technologies have been advanced; image processing as an emerging technology also began to gradually emerge with the sieve guzheng. With the rapid progress of data information analysis and processing technology and robot science in the 21 st century, intelligent automation technology based on machine vision has become the development trend of science and technology, and image processing occupies an increasingly important position as one of the important elements for the realization of intelligent automation. Image retrieval is an indispensable branch of image processing technology, and its complex multi-information fusion processing capability makes it play an important role in huge number of fields such as military, transportation, medical, and aviation remote sensing, so image retrieval technology attracts extensive attention from huge number of domestic and foreign experts and scholars.

Image retrieval techniques can be divided into three main levels: pixel level, feature level, and decision level, with different image features, attributes relying on different levels of retrieval. Pixel-level image retrieval mainly focuses on the pixel point retrieval process, using and preserving the information contained in the source image as much as possible, so the retrieved image is richer in intrinsic information and has more advantages than other levels in terms of accuracy. Feature-level retrieval is more inclined to analyze and process the features of interest in the source image, and selective feature aggregation analysis is achieved by searching for feature information such as contour information, texture information, luminance information, and corner point information, to complete the source image retrieval. Decision-level retrieval is based on cognition, and the decision criteria are formulated by categorizing and analyzing the extracted feature information, and then, the confidence level of each decision is evaluated to make the optimal decision to guide image retrieval. Feature-level retrieval and decision-level retrieval analyze and process the source image features to develop retrieval rules. Compared with pixel-level 
retrieval, these two-level strategies are less computationally intensive and less affected by noise, but the retrieved images lose more information, so they are mostly used in multiple fields such as image recognition, detection and tracking, and image classification. The pixel-level image retrieval characteristics are more closely related to the purpose of information retrieval and therefore have more important research significance sieve, Sasaki. In this paper, we investigate multifocused image retrieval algorithms based on pixel-level retrieval. The backpropagation of the network updates the weight parameters also based on the softmax cross-entropy loss function. The literature [3] improves the differential evolution retrieval method by adding a block expansion selection mechanism while improving the local and global search capability of the algorithm so that the image chunk size can be selected adaptively, thereby reducing the block effect phenomenon of retrieved images. In the literature [4], bootstrap filtering is applied to the field of image retrieval. The method uses differential mean filtering to decompose the source image into approximate layer components and detail layer components and then performs significant analysis on the source image features to obtain the initial weight map. The bootstrap filtering can optimize the initial weight map, and the decision maps of the approximate layer and the detail layer can be obtained by adjusting the relevant parameters, respectively. Finally, the decomposed images are weighted and retrieved according to the decision map to obtain the retrieved images. In literature [5], a multifeature image retrieval technique based on partial differential equations uses image gradient similarity to improve the accuracy of gradient direction and magnitude. The similarity measure of the image is first defined; then, the gradient of the source image is filtered according to the neighborhood similarity of the gradient field, and finally, the retrieved image is obtained by the filtered gradient field. It combines the approximate sparse representation and the improved orthogonal matching tracking algorithm to achieve image retrieval. First, the source image is segmented into multiple small blocks by sliding windows, and then, a fixed number of atoms are set to complete the orthogonal matching iterations thus obtaining the approximate representation of the sparse coefficients, and the approximate sparse coefficients are used to guide the image block retrieval to achieve the retrieved image reconstruction.

The literature [6] converts the multifocus retrieval task into a spot and scatter focus region boundary detection task starting from boundary segmentation, where the source image focus information is segmented out by the detected boundaries of different focus regions, and the regions with different degrees of focus segmented from each source image will be used as the respective retrieval decision maps. For the decision map of multisource images, the region with a higher focus degree is used as a high priority decision to guide the multifocused images to complete the retrieval task. The image retrieval method in the transform domain usually requires the image to be spatially transformed to decompose the source image into multiscale images with different subbands by frequency-domain transformation, and then, the retrieval operations are performed separately for the images of different scales according to specific retrieval rules. The subband images of different scales have different frequency domain characteristics, and the low-frequency subband images reflect the approximate components of the image with high energy, while the high-frequency subbands focus on analyzing the detailed information with gradient features such as image edge contour and detailed texture. Therefore, the transform domain retrieval can be more in line with the visual characteristics of the human eyes, and through the corresponding retrieval process of the captured image features of different spatial scales, the retrieved image can have more significant feature information and detailed texture feature information to meet the effect of human visual perception. At present, the commonly used transform domain retrieval methods are mainly classified into pyramid transform retrieval method, wavelet transforms retrieval method, and multiscale geometric transform method. The literature [7] introduced the Laplace pyramid transform into the field of image retrieval to realize the retrieval of different frequency band components separately and obtain the retrieved image by reconstruction, so it is regarded as the beginning of the transform domain retrieval method. Since then, numerous retrieval methods based on partial differential equations have been proposed one after another.

\section{Multifeature Image Retrieval Technique Based on Partial Differential Equations}

3.1. Partial Differential Equations. In recent years, the methods of variational and partial differential equations, originally from physics and mechanics, have opened a new field in image processing and computer vision, and image processing based on partial differential equations has gained much attention and success. The basic idea is to evolve an image, a curve, or a surface in a partial differential equation model and to obtain the desired result by solving this partial differential equation. The entry of variational and partial differential equation methods into the field of image processing has also undergone a process from scratch, from simple to complex [8]. The image retrieval on the shopping platform is mainly a series of retrievals on graphics. For its characteristics, shape, color, usage classification, etc., users use algorithms to search for its characteristics. Reconciliation finally allows users to use the image recognition technology to identify when users search for pictures and then select products and recommend them to shoppers to improve the efficiency of the shopping platform.

\section{(1) Basic concepts}

The partial differential equation of the function $u(x, y, \cdots)$ is a mathematical relation between the function $u$ and its partial derivatives

$$
F\left(x, y, u, \frac{x 1-\mu 1}{\sigma}, \frac{x 2-\mu 2}{\sigma}, \cdots, \sum_{i=u}^{n} \frac{x i-\mu i}{\sigma}\right)=0
$$


where $F$ is the function, $x, y$ are the independent variables, and $u$ is the response variable. The order of the partial differential equation is the highest number of partial derivatives in the equation. For example, the first-order partial differential equation

$$
F\left(x, y, u, \frac{\delta u}{\delta x}, \frac{\delta y}{\delta u}, \frac{1}{n} \frac{x-\mu}{\sigma}\right)=0 .
$$

Written in arithmetic form as follows:

$$
L_{y} X(u)=f(x) \text {. }
$$

(2) Classical linear partial differential equations

For fluctuation equation, the fluctuation equation is shown in the following equation:

$$
F(u)=\lim _{n \longrightarrow \infty} \sum_{i=1}^{n} X_{i i}\left(X_{1}, \cdots, X_{n}, \frac{1}{n} \sum_{i=1}^{n}\left(X_{i}-\bar{X}\right)^{2}\right) Y n .
$$

(3) On the initial and boundary conditions of partial differential equations, in most cases, the general solution of partial differential equations contains arbitrary functions with some uncertainty, so it is not very meaningful in practice. In this case, some initial conditions or boundary conditions must be attached to get a specific solution. This is the fixed solution problem of the partial differential equation. Fixed solution conditions include initial conditions and boundary conditions. The initial or boundary conditions are usually proposed by a specific problem. The initial condition is Cauchy's condition. There are three types of boundary conditions: Dirichlet condition (the first type of boundary condition), Neumann condition (the second type of boundary condition), and Robin condition (the third type of boundary condition) [9].

(4) Nonlinear partial differential equations

$$
F\left(x, y, u, \frac{\delta y}{\delta x}, \frac{\delta y}{\delta u}, \sum_{i=1}^{n} X_{i}\right)=0
$$

3.2. Variational and Partial Differential Equation Methods in Image Processing. Partial differential equations were introduced into image processing in the 1980s and were well developed in the 1990s. The partial differential equation in the image domain is expressed as

$$
F\left(x, y, i, \frac{d y}{d x}, \frac{\partial^{2} \Omega}{\partial u \partial v}, \frac{1}{n} \frac{\Delta y}{\Delta x}\right)=0 .
$$

(1) Uniqueness of the Model Solution of the Partial Differential Equation. The existence, uniqueness, and stability of the model solution in the image domain [10] can be ensured by setting the initial conditions and diffusion coefficients of the partial differential equation

(2) Local Feature Retention Performance. Partial differential equations process images based on the diffusion of local features and can maintain geometric features such as region boundaries. Partial differential equations can directly model the geometric information such as gradient, curvature, tangent direction, and normal direction in an image, which makes the image visually effective after processing. A large number of different partial differential equation models have been proposed by scientists for different image processing tasks

The main areas where partial differential equations have achieved more significant results in image processing are as follows:

\section{(1) PDE image denoising}

The most classic algorithm in image denoising is the Gaussian low-pass filter, i.e., the thermal diffusion equation. One of the most famous ones is the P-M equation.

$$
F(u)=\lim _{i \longrightarrow \infty} \sum_{i=1}^{n}\left(X_{i}-U\right)^{2}+\lim _{\delta x \longrightarrow 0} \frac{\partial^{2} \Omega}{\partial u \partial v}
$$

\section{(2) PDE image enlargement}

Image interpolation is an important process for superresolution analysis of large images. The partial differential equation achieves interpolation and magnification based on geometric features such as image edges and level set curves. Therefore, partial differential equations can preserve detailed features such as region boundaries, while attenuating noise [11].

\section{(3) PDE image segmentation}

Image segmentation is an important research topic in image processing and computer vision and can be divided into boundary-based segmentation, region-based methods, and hybrid segmentation methods according to the image features used.

3.3. Principle of Multifeature Image Retrieval for Partial Differential Equations. Most of the current research on commodity image retrieval focuses on CBIR, so this paper studies image-based retrieval, whose framework is shown in Figure 1 and consists of three main parts: (1) for the commodity image data set, constructing a feature database by feature extraction model; (2) for the commodity image to be retrieved, obtaining the feature representation of this commodity image by feature extraction model; and (3) matching the fabric image to be retrieved by the (3) match 


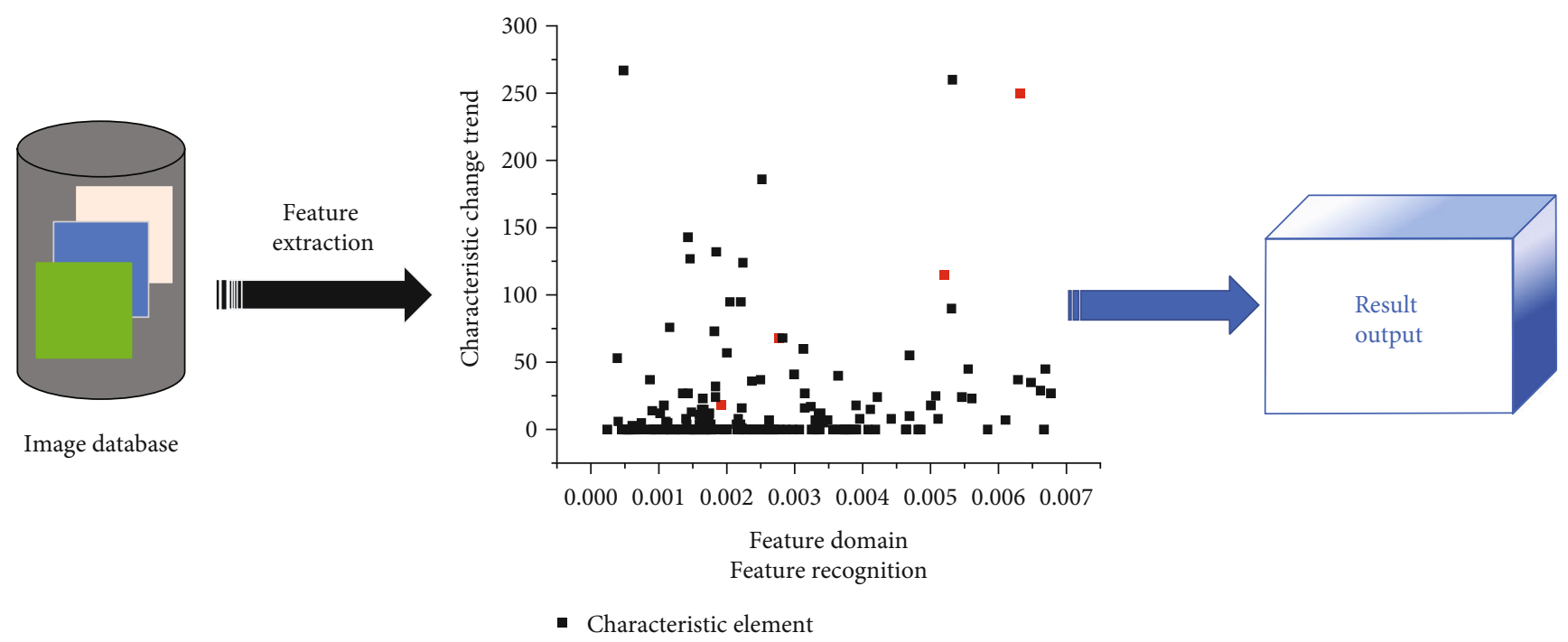

FIGURE 1: Image retrieval framework.

the feature representation of the fabric image to be retrieved with all the feature representations in the feature database, and finally output the result of fabric image retrieval. It involves two key technologies: image feature extraction technology and similarity matching technology [12].

The maturity of the image retrieval model-convolutional neural network (CNN) model has inspired scholars to solve the ideas in image retrieval, and a large number of results have been obtained through a large number of experiments. Using convolutional neural network technology, a deep architecture can be constructed to learn features at different levels from the bottom to the top of the network to represent images, and the feature description of images can be upgraded from the low-level surface visual level to the high-level semantic level by a large number of convolutional operations. The image features extracted by convolutional neural networks are generally referred to as deep features [13].

(1) Multifeatured neural network for partial differential equations

The most basic neural network structure takes the input data, runs it through a series of neurons, and finally outputs it. This model mainly extracts the features of the image. This process has 7 main steps. The features of the image are extracted and finally extracted by computer algorithms and then identified. This is the most important key to the entire process. A partial differential equation convolutional neural network consists of several simple basic neural networks as shown in Figure 2.

The partial differential equation neural network has a strong feature representation capability, except for the input and output layers; the output $y$ of each layer of the network can be used as the depth feature extracted by that layer, which is calculated as follows. This formula is mainly used to identify and extract image features, such as color and shape. The basic principle is that through this formula, some essential characteristics of the image can be seen, so that the algorithm can identify it later.

$$
U K=\sum_{i=1}^{n} X_{i}^{2}, Y K,=\partial(U K-\theta K)+\frac{\partial^{2} \Omega}{\partial v^{2}} .
$$

\section{(2) Activation function}

By introducing nonlinear factors, the feature representation capability of convolutional neural networks can be improved to enable them to solve more complex problems. There are three main activation functions commonly used: the sigmoid function, the tanh function, and the ReLU function. The sigmoid function is calculated as follows [14].

$$
\operatorname{Sigmoid}(x)=\frac{1+e^{x}}{1-e^{x}}+\frac{d y}{d x} \frac{\partial^{2} \Omega}{\partial v^{2}} .
$$

The sigmoid function is an $S$-shaped curve function with a value domain of 0 to 1 . For a certain intermediate layer of a neural network, the smaller the input of the previous layer, the closer the output is to 0 , and the larger the input of the previous layer, the closer the output is to 1 . It takes the input image feature representation and maps it to the output between $(0,1)$, and its output is similar to the working mechanism of a biological neural network, so it can be a good description of the image. The tanh function is formulated as follows.

$$
\tanh (x)=\frac{d y}{d x}+\lim _{\delta x \longrightarrow 0} \frac{\partial^{2} \Omega}{\partial u \partial v} .
$$

The coordinate diagram of the sigmoid function is shown in equation (11), and the function can limit the 


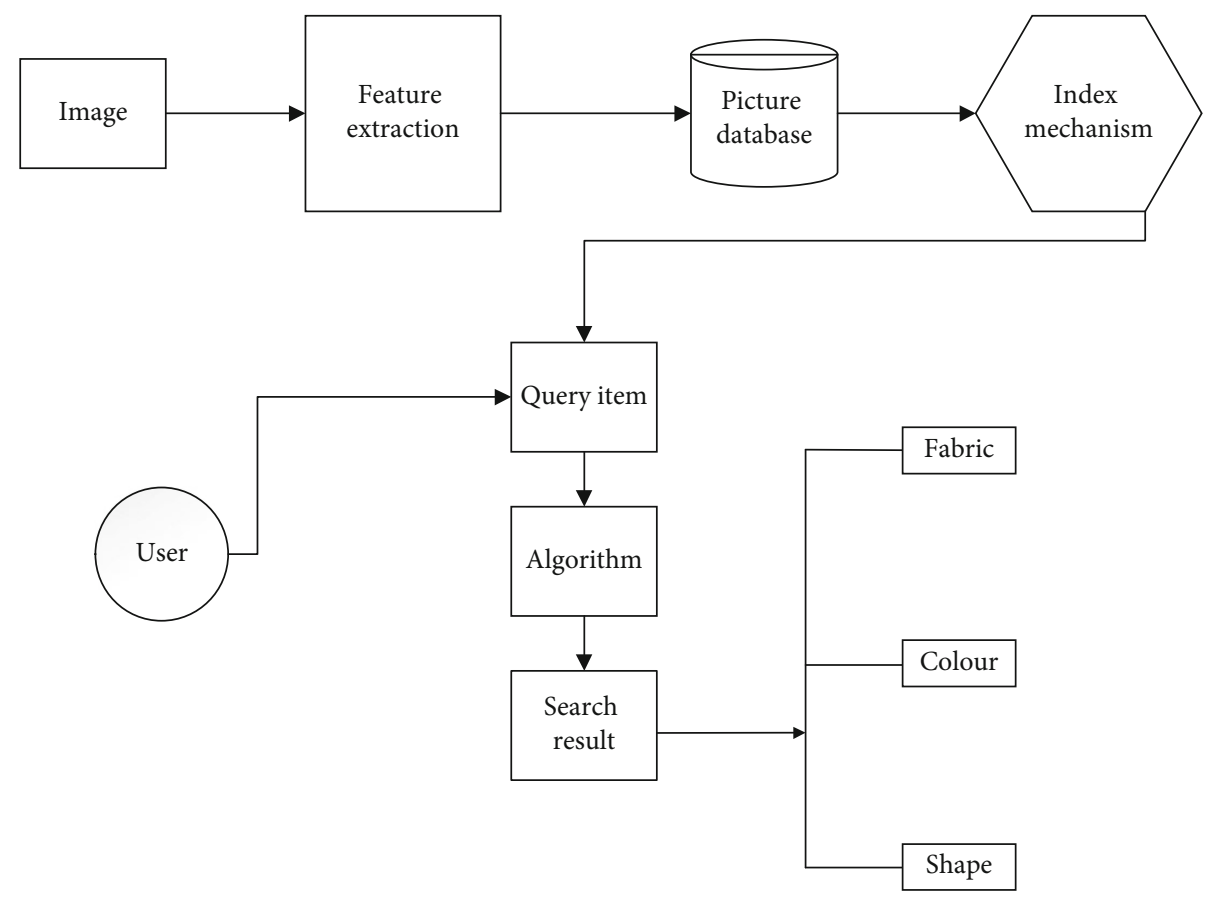

FIgURE 2: Neural network model for partial differential equations.

variation of the larger input values to the interval $[0,1]$. This formula is mainly to identify the basic features of the extracted graphics. The basic principle is that through this formula, some essential characteristics of the image can be seen, so that the algorithm can identify it later.

$$
S(x)=\frac{\partial^{2} \Omega}{\partial u^{2}}+\frac{\partial^{2} \Omega}{\partial v^{2}}+\frac{\partial^{2} \Omega}{\partial u \partial v} .
$$

Tanh function is a deformation of sigmoid function; the difference is that its output value domain is between -1 and 1. Tanh function is centered at 0 , so using the tanh activation function can speed up the training of the model. The function is a hyperbolic tangent function obtained by comparing hyperbolic tangent and cosine functions. Its corresponding equation is as follows.

$$
\tanh (x)=\frac{\csc \theta}{\arcsin \theta}+\lim _{\delta x \longrightarrow 0} \frac{\delta y}{\delta x}
$$

Figure 3 is the coordinate diagram. The data in Figure 3 comes from subsequent experimental data, and there is enough data to support the data graph.

The ReLU function is a segmentation function whose output value is $x$ when $x \geq 0$ and 0 when $x<0$. The ReLU activation function allows the model to converge very quickly and with much higher computational efficiency than other activation functions. Each image feature includes 4096 elements, and the ReLU operation for each element is as follows.

$$
\operatorname{relu}(x)=\frac{2 \cos \theta+e^{i \theta} \sin 2 \theta}{2 \sin \theta}+\frac{\partial^{2} \Omega}{\partial v^{2}}+\frac{\Delta y}{\Delta x}+\frac{d y}{d x}
$$

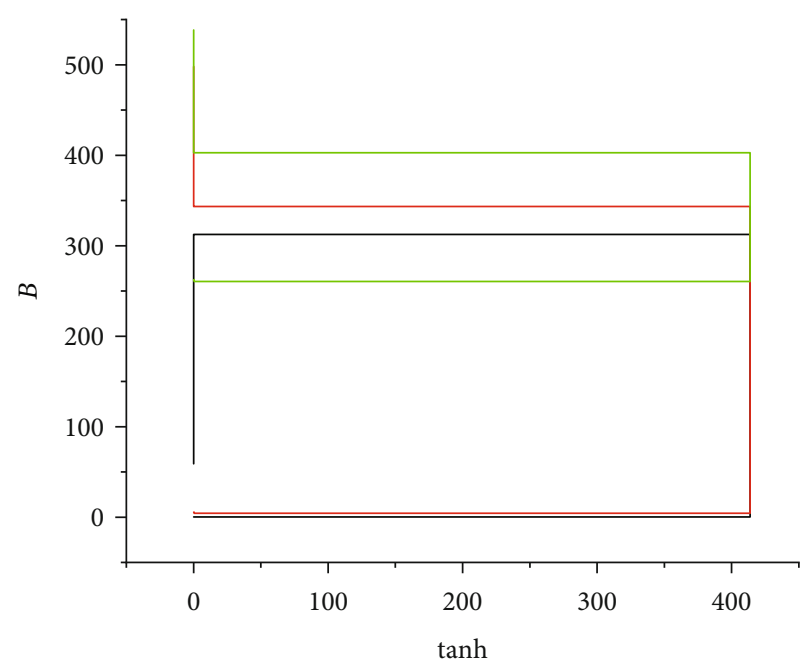

FIgURE 3: The coordinate diagram of tanh function.

ReLU function under the equation.

$$
\operatorname{relu}(x)=\lim _{n \longrightarrow \infty} \frac{1}{n} \sum_{i=1}^{n} X_{i} Y_{i} \sum_{i=1}^{n} U_{i}
$$

Figure 4 is the image of this function. Figure 4 is obviously supported by sufficient data.

The experimental data is reflected in the experimental steps and the experimental process.

The partial differential equation neural network model has shown very powerful performance for tasks related to the field of natural language processing. Researchers have trained the model using the massive text corpus Wikipedia 


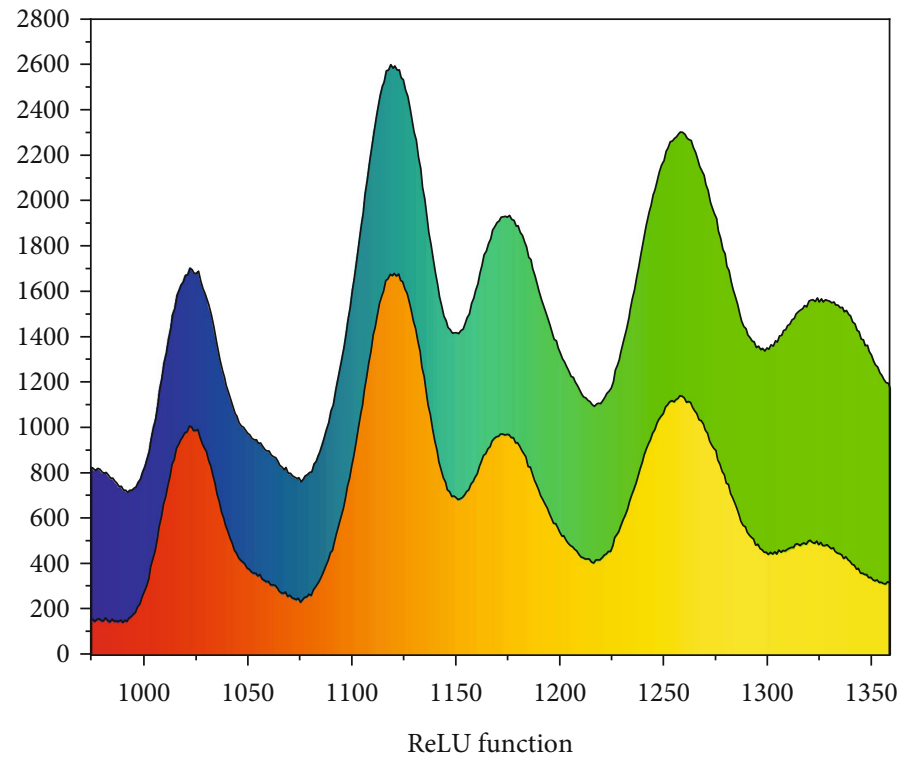

$x \geq 0$
$x<0$

Figure 4: ReLU function.

and books corpus and fine-tuned it for different downstream tasks, so it can be said to be not only a simple language model but also a semantic representation model with generality [15]. Figure 5 is the neural network training effect graph. The training sample in Figure 5 is supported by specific data, which is obtained after a series of tests on the sample.

\section{(3) Algorithms for partial differential equations}

The above model can be trained to correlate image features, and the extracted features are a slack hash code vector, which is still floating-point type, so we follow the general principle of the hash algorithm to speed up image retrieval and convert the slack hash coded feature vector of the fabric image to a binary hash coded feature vector, which is calculated as follows [16].

$$
H\left(x_{i}\right)=\frac{\partial^{2} \Omega}{\partial u \partial v}+\frac{d y}{d x}+X_{1}, \cdots, X_{n}
$$

After training the samples, the fabric image data set is fed into the model to extract the relaxed hash coded feature vectors of the hash coding layer, and then, the relaxed hash coded feature vectors are converted into binary hash coded feature vectors as feature vectors for each image in the data set by the above formula to construct the feature database. Figure 6 is a model retrieval shopping product application.

3.4. Multifeature Image Feature Extraction Technique for Partial Differential Equations. The image search is mainly divided into two parts. One is to train most of the samples in the system to know how to query the product and then to check it through the algorithm. The features of degener- acy are divided into local features and global features. The global features of an image are mainly three types of image color, texture, and shape, while the local features of an image use a part of the image area to extract features. Each of the four types of features is discussed below. The image retrieval method based on traditional machine vision content features represents the visual information of an image with invariant features. The invariant features can cope with the effects of changes in brightness, scale, and orientation to some extent. The invariant features are divided into local features and global features. The global features of an image are mainly three types of image color, texture, and shape, and the local features of an image use a part of the image area to extract features [17].

(1) Color features are the most significant features of an image. For a commodity image, the color of any pixel point is a combination of three original colors (red, green, and blue) by appropriate proportions. Commonly used color features are color histograms and color moments. The color histogram vectorizes the color space without considering the pixel point positions and counts the distribution of each original color in the image. Color moments, on the other hand, do not vectorize the color space but use loworder moments of colors to represent the distribution of each color in the image. The disadvantage of color features is that it is difficult to describe specific objects in the image, and it is impossible to consider their positions [18]

(2) Shape features, which utilize the unique shapes contained in the image for image discrimination, are often used to retrieve specific targets. Shape features 


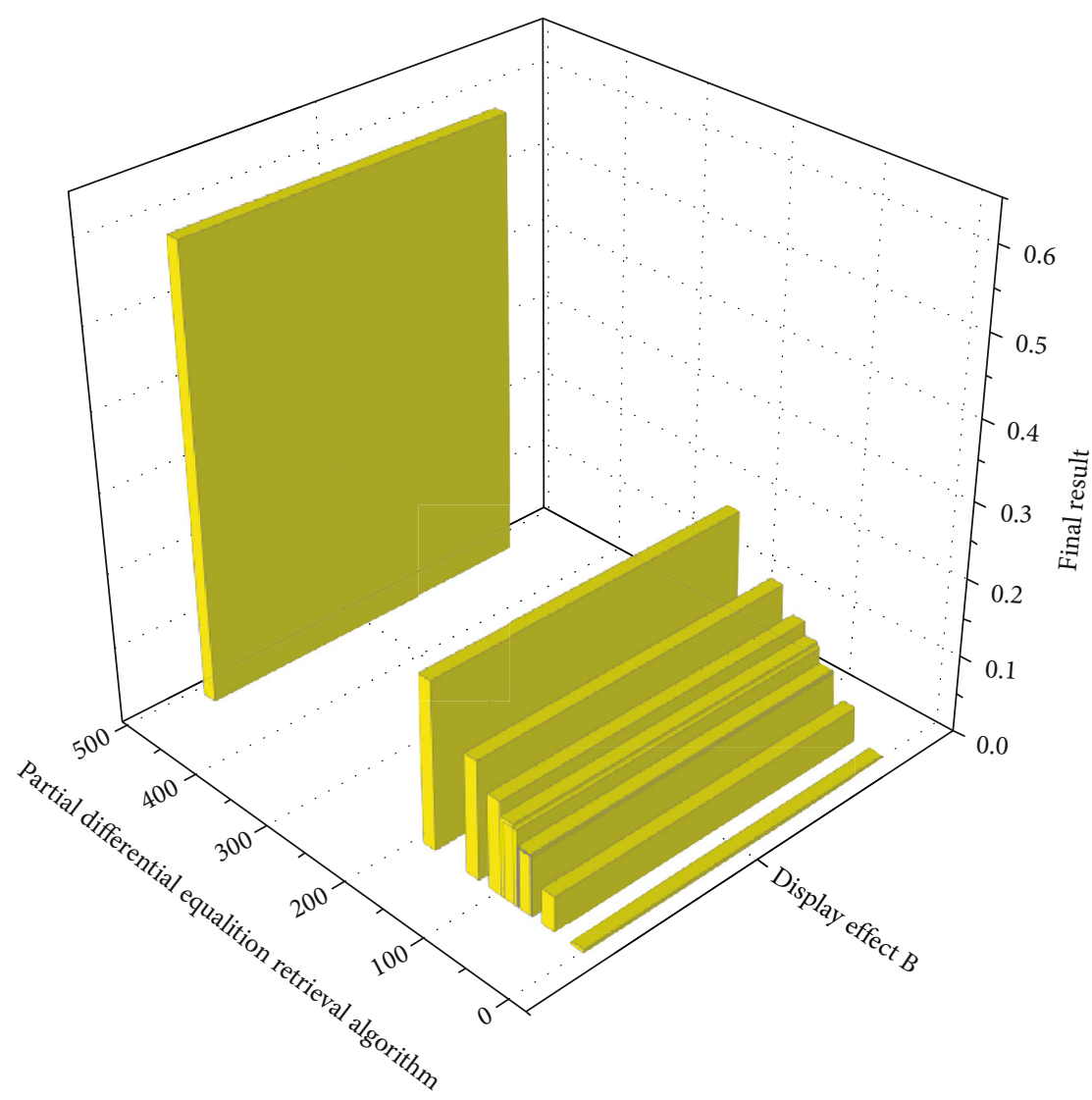

FIgURE 5: Neural network training effect graph.

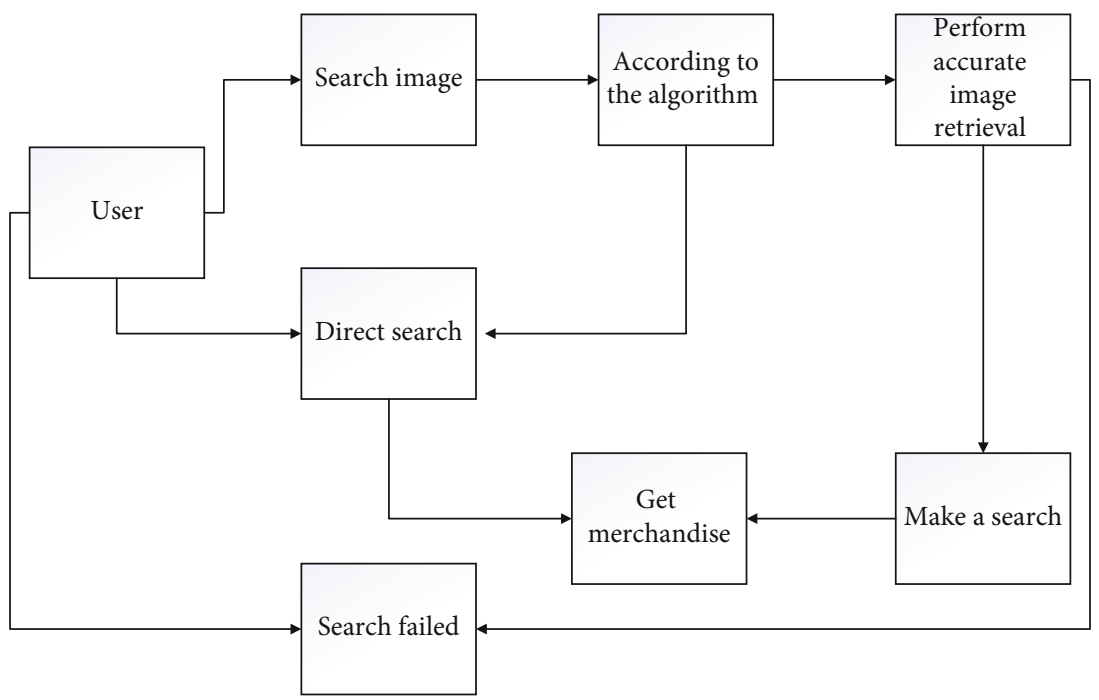

FIgUre 6: Model retrieval shopping product application.

mainly segment graphic elements from images and then extract features based on the segmented key graphics as the basis for discrimination. Shape features can be divided into boundary contour-based shape features and region-based shape features, of which the most common shape features are regionbased invariant moments, which describe the shape of an image by a set of invariant moments. The disadvantage of shape features is the high computational complexity 
(3) Local features, color features, and shape features are global; they are easily affected by factors such as occlusion and displacement in the image, and global features do not have strong differentiation, so local features emerge to represent the features of a certain region in the image. Local features can still represent the features of the region where they exist well when the image is occluded or displaced. And the local features can be combined into global features by different feature aggregation descriptors to represent the features of the whole image more stably. The most commonly used local feature is SIFT, which describes the local features of key points by key point localization. The disadvantages of local features are also obvious, which require very high feature dimensionality to obtain better retrieval results in retrieval and complex computation

3.5. Parameters Related to Multifeature Image Retrieval Model with Partial Differential Equations. With the image feature extraction model, the extracted feature vectors are generally of two types: a floating-point vector and a binary-valued hash-encoded vector that has been dimensionally reduced by a hash-encoding algorithm. For these two types of feature representations, choosing an appropriate similarity matching method can directly improve the efficiency of image identification retrieval [19-21]. Euclidean distance, cosine distance, and Hamming distance are commonly used similarity measures in image identification retrieval Euclidean distance, and cosine distance applies to floating-point vectors, while Hamming distance applies to binary-valued hash-encoded vectors $[22,23]$.

\section{(1) Euclidean distance}

The Euclidean distance is the absolute distance between two image feature vectors in $n$-dimensional space, which is calculated as follows.

$$
\operatorname{dis}(v 1, v 2)=\sqrt{\lim _{n \rightarrow \infty} \sum_{i=1}^{n}\left(X_{i}+Y i\right)}+\frac{x-\mu}{\sigma} \sum_{i=1}^{n} X_{i}^{2} .
$$

\section{(2) Cosine distance}

The cosine distance is calculated by the cosine of the angle between the corresponding feature vectors of the two images, and the smaller the cosine distance is, the more similar the two images are, and it takes the value from 0 to 1 . It is calculated as follows.

$$
\operatorname{dis}\left(v_{1}, v_{2}\right)=\lim _{n \rightarrow \infty}\left(\frac{\sum_{i=1}^{n} X_{i} Y_{i}}{\sqrt{\sum_{i=1}^{n} X_{i}^{2}}}+\frac{1}{n} \sum_{i=1}^{n} X_{i} Y_{i}+\frac{x-\mu}{\sigma}\right) .
$$

(3) Hamming distance

Hamming distance is the statistical number of two images whose binary hash codes correspond to different positions, and if the numbers of the corresponding positions are different, the Hamming distance is added by 1 . The smaller the Hamming distance is, the more similar the corresponding two images are and the closer their binary codes are, and it is calculated as follows.

$$
\operatorname{dis}\left(v_{1}, v_{2}\right)=\lim _{n \longrightarrow \infty}\left(\frac{1}{n} \sum_{i=1}^{n}\left(X_{i}-\bar{X}\right)^{2}+\frac{x-\mu}{\sigma} \sum_{i=1}^{n}\left(X_{i}-\bar{X}\right)^{2}\right)
$$

\section{(4) Metrics}

A metric, also known as a distance function, is understood in the context of mathematics as a function of the distance between the elements in a given set. In the specific process of scientific research, the data utilized are generally correlated with each other, and huge number of excellent algorithms can express these correlations well, although different metrics can vary widely for different data samples. Therefore, a certain metric is often only applicable to a single set or a certain type of data set and does not fully work for all sample data. In the process of calculating feature similarity, the distance function needs to be selected according to the characteristics of the data set, but the selected distance function may not have the expected effect if a certain category of data changes. The metric learning technique avoids this problem by learning a distance function that corresponds to the data when the sample varies, thus completing the task of measuring the distance between the data. Metric learning intends to use the feature vectors of the data after dimensionality reduction to perform distance calculation. Computer data are similar to real-life objects, and there are large or small correlations between each of them. Some classical metric functions often only refer to a single attribute of the data, and the reference sign of the correlation is not obvious. The output of such a calculation, which only considers one attribute and ignores the potential correlation, is not a very effective indication of the distance between the data [20].

For example, the standard equation for the marginal distance is as follows.

$$
d\left(x_{j}, y_{j}\right)=\sqrt{a^{2}+b^{2}} \frac{\Delta y}{\Delta x}+\frac{\partial^{2} \Omega}{\partial v^{2}}+\frac{\delta y}{\delta x}
$$

3.6. Image Retrieval Model for Partial Differential Equations. To extract better depth features, a huge number of convolutional neural network structures with excellent performance have been proposed. Nowadays, the more commonly used convolutional neural networks that can extract image features for image retrieval include AlexNet, VGGNet, InceptionNet, etc. [24, 25]. The basic principles are

$$
\frac{d y}{d x}=\lim _{x \longrightarrow \infty} \sum_{i=1}^{n} X_{i}^{2}+o(\Delta x)
$$




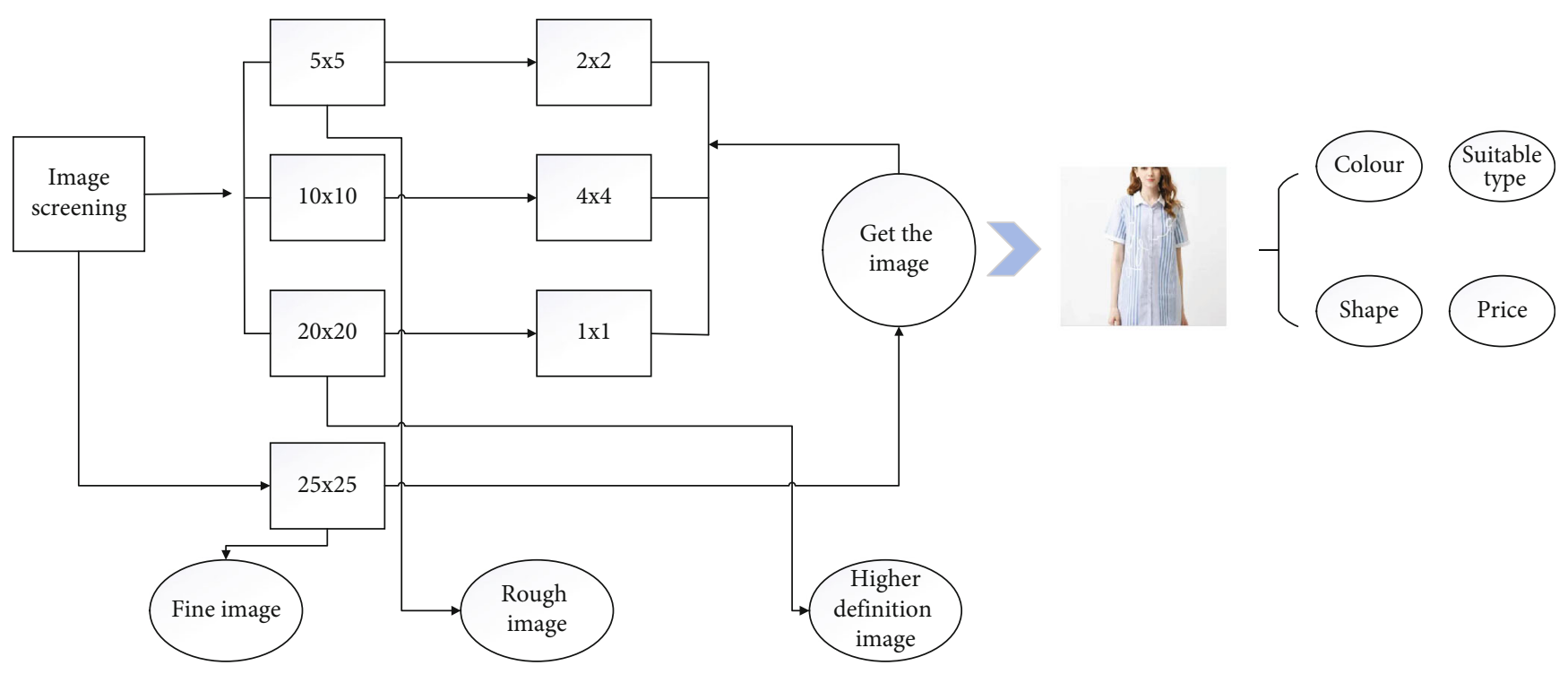

FIGURE 7: Image retrieval model.

AlexNet is a CNN structure with 8 layers, which consists of 5 convolutional layers and 3 fully connected layers. In most image retrieval studies, the output of the penultimate fully connected layer of AlexNet, a 4096-dimensional feature vector, is selected as the feature vector from which the image is extracted. VGGNet is a series of networks with several network layers ranging from 11 to 19 , and in most image retrieval studies, VGGNet16 and VGGNet19 are chosen as the base networks, and the output of the penultimate fully connected layer is generally used as the feature vector extracted from the image. Figure 7 shows the basic structure of InceptionV1, the Inception module, which connects convolutional operations with different scales. $5 * 5,3 * 3$, and $1 * 1$ convolutional kernel output the features of an image as feature representations of different scale sizes. This means that the Inception module fuses features of different scales, and after several consecutive Inception modules, the feature representation of the image fuses features on the lower surface of the image and features on the higher semantic level.

\subsection{Image Retrieval Model Application of Partial Differential} Equations. When we query a pair of images with the multifeature image retrieval technology system using partial differential equations, it can spontaneously match color features with each image in the image library to output a result sorted by similarity when we select color features as the query condition, and it can also match texture features with each image in the image library to have a result output, and when we apply DS evidence theory, we will get a matching result that fuses color, texture, and shape features. The steps of the method for implementing image retrieval with five features extracted by multifeature DS fusion are the following.

(1) The color histogram and color correlation map are used to extract the color features of the query image, respectively; then, the Tamura texture and gray cogeneration matrix are used to extract the texture features of the query image; finally, the Hu moments are used to extract the shape features of the query image; finally, a 115-dimensional vector is obtained to represent the feature vector of the query image

(2) Using the Euclidean distance introduced in the previous section to calculate the similarity distance between the query imageQand the first image color in the image library, $21 \mathrm{mmd}$ and the similarity distance between the query imageQand the first image texture in the image library, $43 \mathrm{mmd}$ and the similarity distance between the query imageQand the first image shape in the image library, and $5 \mathrm{md}$, respectively, the 10 most similar images are returned and the finding accuracy of single feature retrieval formula. Figure 8 is image retrieval application flow

(3) In this paper, we use the similar distance of color features, $21 \mathrm{mmd}$ to determine the color trust of each image and the texture trust of each image based on the similar distance of texture features, $43 \mathrm{mmd}$ and similarly, and $5 \mathrm{md}$ to determine the trust of shape features

(4) Since each vector element in the feature vectors of texture features and color features and shape features represents a different physical meaning, the feature vector normalization process is performed first, followed by the orthogonal calculation

(5) The trust degree of all five features of each image is obtained and then multiplied by their respective weights, and the total trust degree of each image is calculated according to the formula

(6) Based on the accuracy of the returned images and removing the features with the lowest single feature 


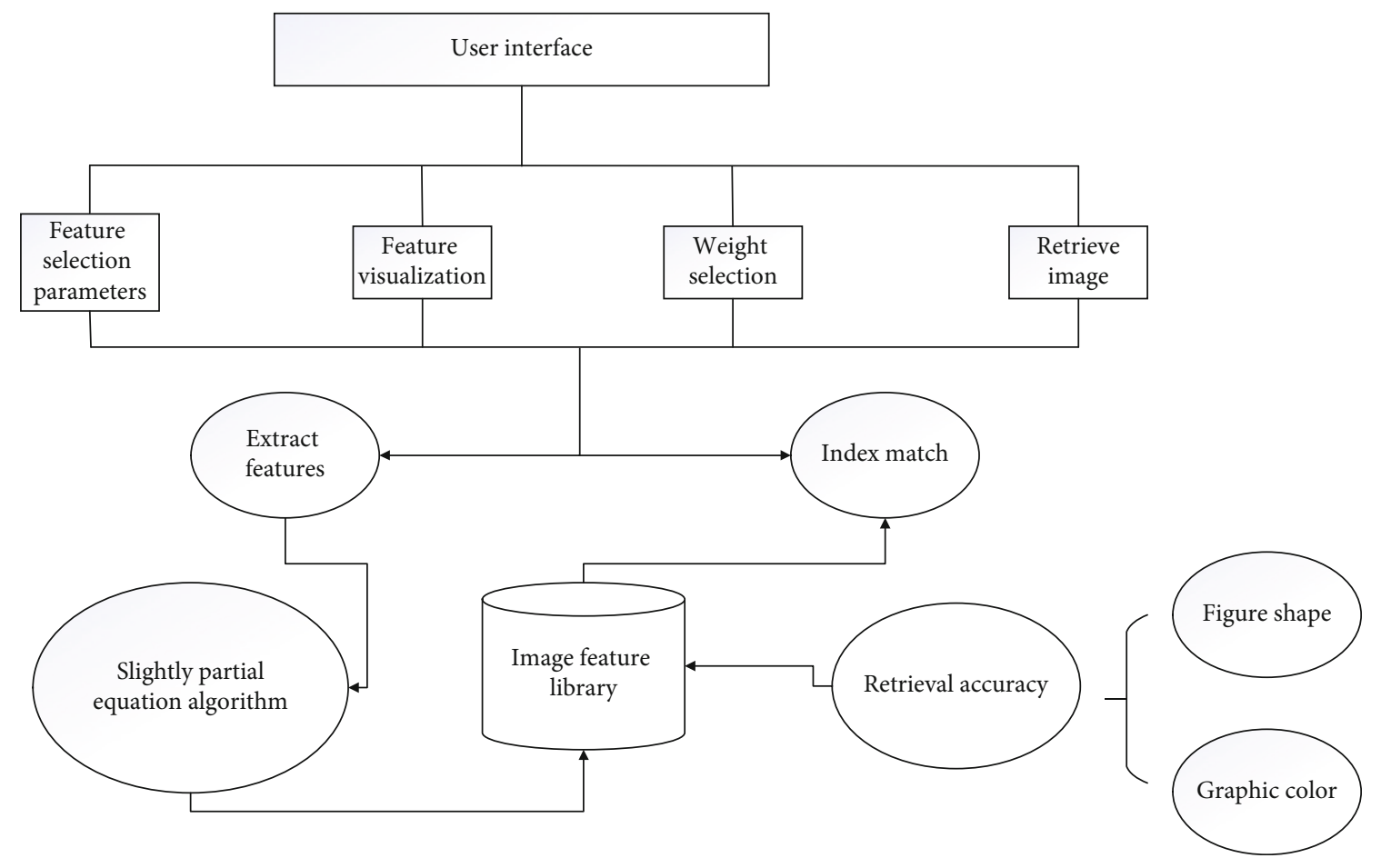

FIGURE 8: Image retrieval application flow.

TABLE 1: Comparison of the average search rate of the two search methods.

\begin{tabular}{|c|c|c|c|c|c|}
\hline Image category & Africa & Beaches & Monuments & Buses & Horses \\
\hline Linear search & $90.70 \%$ & $90.20 \%$ & $99.30 \%$ & $90.50 \%$ & $98.70 \%$ \\
\hline Algorithm search & $96.90 \%$ & $97.20 \%$ & $66.40 \%$ & $87.00 \%$ & $98.80 \%$ \\
\hline Algorithm search & $99.90 \%$ & $99.20 \%$ & $99.25 \%$ & $98.78 \%$ & $87.20 \%$ \\
\hline
\end{tabular}

checking rate, the total trust of each image is recalculated by the formula, and the most similar image requested by the user is returned

\section{Experimental Results and Analysis}

Thirty images from each category were selected as examples, and the query examples were retrieved using linear search and partial differential equation-based multifeatures based on color histogram features. The top 20 average search accuracy and average response time of the search results were calculated. The average search rate of the two search methods is shown in Table 1.

For these five types of images, in terms of retrieval accuracy, the average search accuracy obtained using linear retrieval is higher than that of Kd-tree retrieval for the other four categories, except for the buses category, which is slightly lower than that of Kd-tree retrieval. From Table 2, it is clear that the response time for the nearest neighbor query using Kd-tree is much less than that of a linear search. The images in the image library are selected for querying, and the methods proposed in the above section on retrieving color and texture features are selected, i.e., color histogram features, gray-gradient covariance matrix features, and basic
TABLE 2: Comparison of the average corresponding time of the two search methods.

\begin{tabular}{lccc}
\hline Search method & Linear search & \multicolumn{2}{c}{ Algorithm search } \\
Build an & $\begin{array}{c}\text { Nearest } \\
\text { index tree }\end{array}$ & neighbor query \\
\hline Responsive practice & $0.009 \mathrm{~s}$ & $0.002 \mathrm{~s}$ & $0.001 \mathrm{~s}$ \\
Response status & Fast & Fast & Speed \\
\hline
\end{tabular}

LBP features are selected to describe the image content, and the three feature weights are in the ratio of $4: 1: 3$. The retrieval results are used as user feedback objects for users to mark whether they are relevant or not. For feedback, method III uses the IGSO-SVM method for feedback. Figure 9 is a graph of experimental results of two ways.

From the five categories of images selected from the image library, namely, Africa, beaches, monuments, buses, and horses, the following analysis can be drawn from the experimental results.

(1) The advantages and disadvantages of the five different feature extraction methods used in this experiment are compared and analyzed, and the 


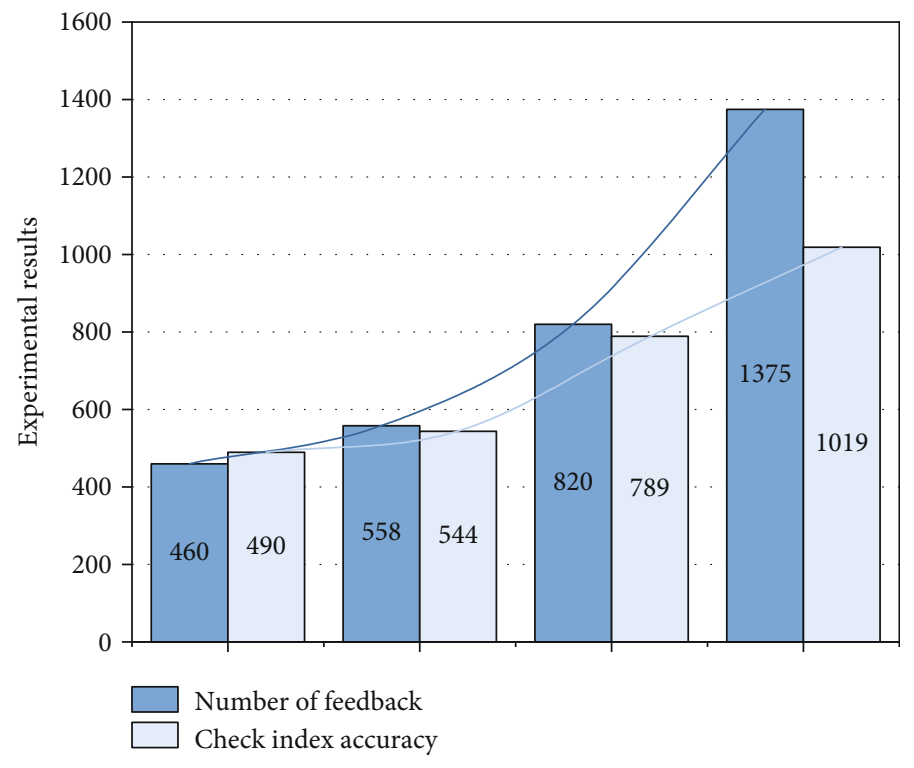

FIGURE 9: Graph of experimental results of two ways.

differences between image retrieval algorithms based on single features and those based on simple multifeature fusion are compared and analyzed. The experimental results show that for the same class of images, the performance of different feature extraction methods is different; for example, for the indigenous class of images, the detection accuracy of the histogram is 0.67 and that of the invariant moment is 0.11 ; for the snowy mountain class of images, the detection accuracy of the histogram is 0.11 and that of the invariant moment is 0.33

(2) The image retrieval algorithm of weighted multifeatures based on the proportion of the search accuracy is proposed based on the analysis and comparison of the search accuracy of single features obtained in Section 2. The experimental results show that compared with the equiproportional multifeature retrieval, the detection rate is improved by nearly $20 \%$ for both indigenous and snow mountain images, while the detection rate is reduced by $7 \%$ for dinosaur images. Based on this, a weighted multifeature image retrieval algorithm based on DS theory was constructed, and it was demonstrated that the accuracy of this algorithm was improved for all categories of images compared to equal scale multifeature retrieval, and the accuracy of dinosaur images was improved to 0.9

(3) In this paper, a multifeature image retrieval technique based on partial differential equations is constructed. The experimental results show that the average accuracy of 0.66 and 0.71 improves to 0.85 for all categories of images compared to the first two image retrieval algorithms which are entirely content-based, indicating that it has better retrieval capability than the first 2 algorithms. Finally, because of the limitations of the authors' level and the lack of time, etc., the research in this paper has huge number of imperfections. In future work, we will further improve the technique of multifeature image retrieval based on partial differential equations and further investigate other algorithms based on semantic aspects. The algorithm proposed in this paper is effective for user clustering in web access patterns, which has been proved on the experiments of the data set, and is not well applied in the mining of data due to the shortcomings of traditional image retrieval techniques, while the present algorithm improves the shortcomings of traditional image retrieval techniques and applies them to the mining of Weblog data, which provides the possibility of personalized design for business websites and has broad application prospects. Further work is to extend this algorithm by combining user registration information, such as age, gender, income, and region, with access periods

From the experimental results, it can be seen that the improved multifeatured image retrieval algorithm with partial differential equations can obtain more suitable classifier parameters, which makes the IGSO-SVM-based correlation feedback method more capable of image retrieval in online shopping and improve people's online shopping experience than the unimproved algorithm without affecting the retrieval performance.

\section{Conclusion}

In this paper, firstly, by understanding the current application status of multifeature image retrieval technology and the existing feature extraction methods, an improved weighted multifeature image retrieval algorithm introducing 
partial differential equation theory is further proposed based on image retrieval technology; meanwhile, a preliminary attempt is made in semantic-based image retrieval. The research of multifeature image retrieval technology based on partial differential equations has been explored. It has been applied in a lot of domain ranges, but it has not been much applied in this e-commerce shopping platform with strong commodity visual dependence. This paper points out the feasibility and urgency of its application in shopping platform based on the analysis of the characteristics of content-based image retrieval technology, as well as the background processing of commodity images to achieve image retrieval matching and the process of commodity retrieval matching, through this new technology. Through the application of this new technology, the "information island" effect can be eliminated to a large extent, the unfairness of competition among merchants can be eliminated, and the situation that the search does not meet the demand due to the incompleteness of consumer information can be reduced, while personalized services can be provided to end users; the customer base can be expanded, and interactive services can be promoted on e-commerce platforms, which can to a certain extent; it can promote faster and better development and innovation of e-commerce shopping platform and can better promote online shopping. This algorithm is still under experimentation and has not yet been used in the market, so further training of samples and improvements to the algorithm are needed.

\section{Data Availability}

The data used to support the findings of this study are available from the corresponding author upon request.

\section{Conflicts of Interest}

The authors declare that they have no known competing financial interests or personal relationships that could have appeared to influence the work reported in this paper.

\section{Acknowledgments}

This work was supported by doctoral fund project of Shandong Jianzhu University: The Effects of Word-ofMouth Information Characteristics on Consumer Confusion (X19075Z0101).

\section{References}

[1] K. M. Owolabi, A. Atangana, and A. Akgul, "Modelling and analysis of fractal-fractional partial differential equations: application to reaction-diffusion model," Alexandria Engineering Journal, vol. 59, no. 4, pp. 2477-2490, 2020.

[2] A. Atangana and S. İğret Araz, "Extension of Atangana-Seda numerical method to partial differential equations with integer and non-integer order," Alexandria Engineering Journal, vol. 59, no. 4, pp. 2355-2370, 2020.

[3] V. S. Parvathy and S. Pothiraj, "Multi-modality medical image fusion using hybridization of binary crow search optimiza- tion," Health Care Management Science, vol. 23, no. 4, pp. 661-669, 2020.

[4] A. R. Alharbi and M. B. Almatrafi, "Riccati-Bernoulli subODE approach on the partial differential equations and applications," International Journal of Mathematics and Computer Science, vol. 15, no. 1, pp. 367-388, 2020.

[5] C. Tian, K. C. Chang, and J. S. Chen, "Application of hyperbolic partial differential equations in global optimal scheduling of UAV," Alexandria Engineering Journal, vol. 59, no. 4, pp. 2283-2289, 2020.

[6] X. Lü and S. J. Chen, "Interaction solutions to nonlinear partial differential equations via Hirota bilinear forms: one-lumpmulti-stripe and one-lump-multi-soliton types," Nonlinear Dynamics, vol. 103, no. 1, pp. 947-977, 2021.

[7] L. Zhu, X. Lu, Z. Cheng, J. Li, and H. Zhang, "Deep collaborative multi-view hashing for large-scale image search," IEEE Transactions on Image Processing, vol. 29, pp. 4643-4655, 2020.

[8] H. Dong, B. Zou, L. Zhang, and S. Zhang, "Automatic design of CNNs via differentiable neural architecture search for PolSAR image classification," IEEE Transactions on Geoscience and Remote Sensing, vol. 58, no. 9, pp. 6362-6375, 2020.

[9] Y. Tsushima, H. Hirasawa, Y. Wakabayashi et al., "Possible solution for the problem of unread image interpretation reports: the "Gunma University Star Search"," Japanese Journal of Radiology, vol. 38, no. 7, pp. 643-648, 2020.

[10] G. Yuan, J. Lu, and Z. Wang, "The modified PRP conjugate gradient algorithm under a non-descent line search and its application in the Muskingum model and image restoration problems," Soft Computing, vol. 25, no. 8, pp. 5867-5879, 2021.

[11] A. Senthilselvi, R. Sukumar, and S. Senthilpandi, "Hybrid fuzzy logic and gravitational search algorithm-based multiple filters for image restoration," International Journal of Data Analysis Techniques and Strategies, vol. 12, no. 1, pp. 76-97, 2020.

[12] J. Yu, A. Kasim, J. Sreenivasan, H. Dzakiria, and A. Ul Haq Magray, "Exploring destination image, familiarity, information search behaviour, involvement and travel motivation as influencers of ecotourists' destination loyalty," Turyzm/Tourism, vol. 30, no. 2, pp. 101-114, 2020.

[13] A. Ovtchinnikova, "Shadows of forgotten ancestors: in search of national representation in the image of folklore," Studies in Costume \& Performance, vol. 6, no. 1, pp. 49-68, 2021.

[14] J. Zheng, W. Song, Y. Wu, and F. Liu, "Image interpolation with adaptivek-nearest neighbours search and random nonlinear regression," IET Image Processing, vol. 14, no. 8, pp. 1539-1548, 2020.

[15] R. N. N. Naseri, "What is a population in online shopping research? A perspective from Malaysia," Turkish Journal of Computer and Mathematics Education (TURCOMAT), vol. 12, no. 4, pp. 654-658, 2021.

[16] G. Xi, X. Cao, and F. Zhen, "The impacts of same day delivery online shopping on local store shopping in Nanjing, China," Transportation Research Part A: Policy and Practice, vol. 136, pp. 35-47, 2020.

[17] Faculty of Business Administration, Banking University and V. D. TRAN, “The relationship among product risk, perceived satisfaction and purchase intentions for online shopping," The Journal of Asian Finance, Economics, and Business, vol. 7, no. 6, pp. 221-231, 2020. 
[18] A. Bhatti and S. Ur Rehman, "Perceived benefits and perceived risks effect on online shopping behavior with the mediating role of consumer purchase intention in Pakistan," International Journal of Management Studies, vol. 26, no. 1, pp. 33$54,2020$.

[19] S. Y. Ahmed, B. J. Ali, and C. Top, "Understanding the impact of trust, perceived risk, and perceived technology on the online shopping intentions: case study in Kurdistan Region of Iraq," Journal of Contemporary Issues in Business and Government, vol. 27, no. 3, pp. 2136-2153, 2021.

[20] F. Rasty, S. H. Mirghafoori, S. Saeida Ardekani, and P. Ajdari, "Trust barriers to online shopping: investigating and prioritizing trust barriers in an intuitionistic fuzzy environment," International Journal of Consumer Studies, vol. 45, no. 5, pp. 1030-1046, 2021.

[21] S. A. Carrillo, "Summability in a monomial for some classes of singularly perturbed partial differential equations," Publicacions Matemàtiques, vol. 65, no. 1, pp. 83-127, 2021.

[22] A. Ali, A. A. Abdullah, and A. Ahmad, "The solution of Poisson partial differential equations via Double Laplace Transform Method," Partial Differential Equations in Applied Mathematics, vol. 4, p. 100058, 2021.

[23] E. Abad-Segura, M. E. Morales, F. J. Cortés-García, and L. J. Belmonte-Ureña, "Industrial processes management for a sustainable society: global research analysis," $P R O$, vol. 8 , no. 5, p. 631,2020

[24] M. Griebel, H. Harbrecht, and M. D. Multerer, "Multilevel quadrature for elliptic parametric partial differential equations in case of polygonal approximations of curved domains," SIAM Journal on Numerical Analysis, vol. 58, no. 1, pp. 684705, 2020.

[25] B. S. Han and K. H. Kim, "Boundary behavior and interior Holder regularity of the solution to nonlinear stochastic partial differential equation driven by space-time white noise," Journal of Differential Equations, vol. 269, no. 11, pp. 9904-9935, 2020. 